\title{
Gastric amyloidosis presenting as acute upper gastrointestinal bleeding: a case report
}

\author{
Rachael Chan ${ }^{1 *}$ and Stephanie Carpentier ${ }^{1,2}$
}

\begin{abstract}
Background: Amyloidosis is characterized by extracellular tissue deposition of fibrils, composed of insoluble lowmolecular-weight protein subunits. The type, location, and extent of fibril deposition generates variable clinical manifestations. Gastrointestinal (GI) bleeding due to amyloid deposition is infrequent. Previous literature describes upper Gl bleeding (UGIB) in patients with known amyloid disease. Here, we describe a case of recurrent UGIB that ultimately led to a diagnosis of $\mathrm{Gl}$ amyloidosis and multiple myeloma in a patient with no history of either.

Case presentation: A 76-year-old male presented to the emergency department with frank hematemesis, melena, and a decreased level of consciousness. Management required intensive care unit (ICU) admission with transfusion, intubation, and hemodynamic support. Upper endoscopy revealed gastritis with erosions and nodularity in the gastric cardia and antrum. Hemostasis of a suspected bleeding fundic varix could not be achieved. Subsequently, the patient underwent computerized tomography (CT) angiography and an interventional radiologist completed embolization of the left gastric artery to address potentially life-threatening bleeding. Complications included development of bilateral pleural effusions and subsegmental pulmonary emboli. Pleural fluid was negative for malignancy. He was transferred to a peripheral hospital for continued care and rehabilitation. Unfortunately, he began re-bleeding and was transferred back to our tertiary center, requiring re-admission to the ICU and repeat endoscopy. Repeat biopsy of the gastric cardial nodularity was reported as active chronic gastritis and ulceration. However, based on the unusual endoscopic appearance, clinical suspicion for malignancy remained high. He exhibited symptoms of congestive heart failure following standard resuscitation. Transthoracic echocardiogram (TTE) demonstrated a reduced ejection fraction of $35-40 \%$ and a strain pattern with apical sparing. Following discussions between the treating gastroenterologist, consulting cardiologist, and pathologist, Congo Red staining was performed, revealing submucosal amyloid deposits. Hematology was consulted and investigations led to diagnosis of multiple myeloma (MM) and immunoglobulin lightchain (AL) amyloidosis. The patient was treated for MM for four months prior to cessation of therapy due to functional and cognitive decline.
\end{abstract}

Conclusions: Gl amyloidosis can present with various non-specific clinical symptoms and endoscopic findings, rendering diagnosis a challenge. This case illustrates $\mathrm{Gl}$ amyloidosis as a potential—albeit rare—etiology of UGIB.

Keywords: Upper Gl bleeding, Amyloidosis, Case report

*Correspondence: rachael.chan@dal.ca

1 Department of Medicine, Dalhousie University and Nova Scotia Health, QEIl Health Sciences Centre, VG Site, Suite 442 Bethune Building, 1276

South Park Street, Halifax, NS B3H 2Y9, Canada

Full list of author information is available at the end of the article

\section{Background}

Upper gastrointestinal bleeding (UGIB) is a common cause of emergency department visits and hospital admissions. Peptic ulcer disease, gastritis, and esophagitis have shared risk factors and are the most common causes of UGIB, accounting for $79 \%$ of cases [1]. 
Amyloidosis is characterized by extracellular deposition of fibrils, composed of insoluble low-molecularweight protein subunits [2]. There are many forms of amyloidosis, classified based on the origin of the deposited protein. Clinical manifestations depend on the type, location, and extent of fibril deposition. Gastrointestinal (GI) amyloidosis can present with non-specific symptoms and endoscopic findings, causing diagnostic difficulty [3]. There have been case reports of UGIB from amyloid deposition in the setting of a known diagnosis of multiple myeloma (MM) [4-6]. We present a case of UGIB, with subsequent investigations leading to the diagnosis of gastric light-chain (AL) amyloidosis and MM.

\section{Case presentation}

A 76-year-old male with coronary artery disease, hypertension, dyslipidemia, and gout presented to hospital with frank hematemesis, melena, and a decreased level of consciousness. He denied any alcohol intake and was a previous smoker. Physical exam was non-contributory other than his decreased level of consciousness. He was admitted to the intensive care unit (ICU), requiring intubation, transfusion, and vasopressor support.

Esophagogastroduodenoscopy (EGD) revealed diffuse gastritis, as well as nodularity and ulceration of the cardia. Hemostasis of what appeared to be a bleeding fundic varix was not performed due to potential diagnostic uncertainty. Subsequent computerized tomography (CT) angiography was completed for clarification, which showed contrast extravasation from the left gastric artery. Given that hemostasis was not achieved on index endoscopy, radiologic evidence of bleeding, and the patient's critical condition, the treating interventional radiologist performed embolization of the left gastric artery. Imaging did not suggest portal hypertension but showed irregularity along the gastric cardia and fundus. Initial biopsies were reported as chronic gastritis, negative for H.pylori or malignancy.

Complications during index admission included development of bilateral subsegmental pulmonary emboli and bilateral pleural effusion. Treatment included therapeutic anticoagulation and bilateral thoracentesis, with pleural fluid analysis consistent with a transudative effusion, negative for malignancy. The patient developed symptoms of significant congestive heart failure following standard resuscitation. Chest radiograph showed vascular congestion. Transthoracic echocardiogram (TTE) showed a strain pattern with apical sparing, moderate left ventricular systolic dysfunction, and an ejection fraction of $35-40 \%$. Repeat endoscopy was arranged to clarify findings and revealed persistent nodularity and ulceration of the cardia (Fig. 1). The patient's suggestive cardiac manifestations and unexplained appearance on EGD led to

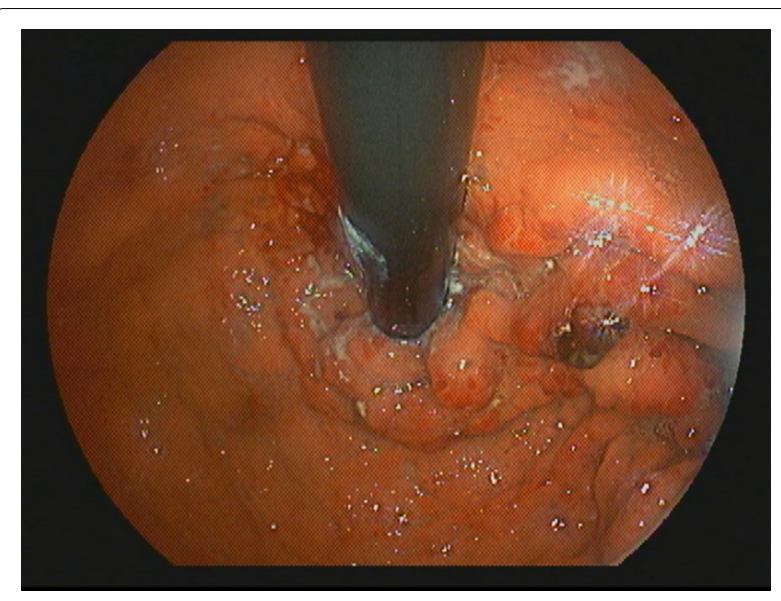

Fig. 1 Full view of gastric cardia showing nodularity, edema, and surrounding ulceration

case discussion between treating gastroenterologist, consulting cardiologist, and pathologist, prompting staining of gastric biopsies for amyloid. In the interim, the patient was transferred to a peripheral hospital for continued care and rehabilitation prior to results being available.

Unfortunately, the patient developed signs and symptoms of recurrent UGIB, warranting transfer back to our tertiary hospital for re-admission to the ICU. EGD on this occasion revealed a duodenal ulcer with a visible vessel which was treated with 1:10,000 epinephrine injection and cautery. Hemostasis was achieved. It was at this point that prior gastric biopsy results became available. Congo Red staining revealed apple green birefringent material under polarized light, consistent with amyloid deposition.

Subsequent investigations during this re-admission revealed elevated serum kappa light-chains and an elevated kappa/lambda ratio. Serum protein electrophoresis showed an abnormal $3 \mathrm{~g} / \mathrm{L}$ band of IgG kappa. A diagnosis of multiple myeloma was made. The patient was followed by hematology and actively treated with cyclophosphamide, bortezomib, and dexamethasone (CyBorD) treatment protocol for four months. Despite no recurrence of GI bleeding, the patient experienced progressive weakness, functional and cognitive decline, and was ultimately unable to tolerate further chemotherapy. Subsequently, based on the patient's goals of care, curative therapy was suspended, comfort care was prioritized, and the patient passed away peacefully.

\section{Discussion and conclusions}

Amyloidosis can be acquired or genetic and is classified based on etiology of the deposited insoluble lowmolecular-weight protein subunits. The most common 
forms of acquired amyloidosis are AL, as in our case; serum amyloid A (AA), which is an acute phase reactant and complication of chronic inflammatory disease; and $\beta-2$ microglobulin, which is dialysis related with protein accumulation due to decreased renal clearance. The continuous protein deposition and build-up damages the integrity and function of affected organs.

AL amyloidosis is caused by plasma cell dyscrasia and deposition of immunoglobulin light chains. Determining the epidemiology of AL amyloidosis is difficult due to its rarity. AL amyloidosis is also challenging to diagnose, given its various presenting clinical syndromes. American data reported in 2018 suggests the incidence and prevalence of AL amyloidosis is between 9.7 and 14.0 cases per million person-years and 40.5 cases per million, respectively [7].The majority of patients with AL amyloidosis are over 65-years-old. The most common clinical presentations of AL amyloidosis are nephrotic syndrome and restrictive cardiomyopathy [8]. Tissue biopsy stained with Congo Red showing green birefringence under polarized light is the diagnostic gold standard [2]. The sensitivity and specificity of biopsy location is variable and may involve abdominal fat pad aspiration or biopsy of the organ in which amyloid is suspected, acknowledging increased risks of hemorrhage with the latter. Rectal biopsy is another surrogate site utilized to diagnose systemic amyloidosis with a reported sensitivity of $85 \%$ but is no longer recommended as a first-line option for diagnosis due to patient discomfort, risk of complications, and low diagnostic yield in the setting of negative fat pad aspiration [9].

Treatment of AL amyloidosis aims to reduce the production of fibrils to limit deposition-and therefore damage-within amyloidogenic organs. There are many therapeutic regimens used to treat $\mathrm{AL}$ amyloidosis, but lack of randomized data comparing efficacy makes decisions surrounding therapy difficult. Since the underlying pathophysiology is plasma cell dyscrasia, management has similarities to that of MM. However, outcomes are better in AL amyloidosis than MM. Treatment options include autologous stem cell transplant (ASCT) and various chemotherapy regimens such as: melphalan and dexamethasone (MDex), cyclophosphamide, thalidomide and dexamethasone (CTDa), and CyBorD [2].

We performed a review of the literature regarding $\mathrm{AL}$ amyloidosis in the GI system. PubMed database was used. No date restrictions were used. Only English-language reports were included. Case report, retrospective chart review, and previous systemic reviews that discussed presentation, diagnosis, and management of gastrointestinal amyloidosis were included. There exists no higher-level evidence. Search Terms used: "amyloid", "AL amyloidosis" "amyloidosis", "gastrointestinal amyloidosis", "endoscopy amyloidosis". 31 results were obtained and formed the basis for this focused review. Articles were further selected based on relevance.

Previous retrospective chart reviews suggest that Gl tract involvement is uncommon in AL amyloidosis, with biopsy proven [10] and clinically apparent [11] disease in $3 \%$ and $1 \%$ of patients, respectively. The majority of patients with GI amyloid have systemic involvement [10]. There are various GI manifestations of AL amyloidosis, including weight loss, GI bleeding, heartburn, and nausea [10]. Common sites of AL amyloid infiltration include the duodenum, stomach, colorectum, and esophagus [3, $10,12]$.With regards to endoscopic abnormalities in those with AL amyloidosis and GI symptoms, 50\% present with GI bleeding secondary to ischemia, vascular friability, or mucosal/submucosal lesions [13]. Endoscopic appearances of GI manifestations of AL amyloidosis are highly variable, differ based on anatomic location, and are not specific to amyloidosis subtype [3]. The most common gastric findings include depressed lesions (45\%), erythema (32\%), edema (29\%), mucosal friability $(26 \%)$, and elevated lesions similar to submucosal tumors (26\%); conversely, there may be no gastric findings (45\%) [3].

Therapy for GI manifestations of AL amyloidosis are primarily supportive including nutritional supplementation and treatment of diarrhea or obstruction [14].There are no treatment guidelines for endoscopic management of UGIB secondary to AL amyloid disease [9]. Based on a prospective cohort, the prognosis of those with GI manifestations of AL amyloidosis is worse compared to those without GI sequelae, with median survival reported as 7.95 months and 15.84 months, respectively [15].

Recent case reports describe UGIB in patients with known multiple myeloma or monoclonal gammopathy of unknown significance $[16,17]$. This case differs because the patient lacked a prior diagnosis and his presentation of recurrent UGIB led to the discovery of his underlying AL amyloidosis. Advanced and irreversible organ dysfunction often precedes the diagnosis of amyloidosis [2]. Therefore, a high index of suspicion and an accessible target organ for diagnostic examination are of utmost importance in expediting treatment and management. Overall, GI amyloidosis presenting as recurrent UGIB is a rare, but known manifestation of AL amyloid disease. This case highlights GI amyloidosis as a differential diagnostic consideration for UGIB and provides an additional example of its endoscopic appearance.

\section{Abbreviations}

Gl: Gastrointestinal; UGIB: Upper gastrointestinal bleeding; AL: Light chain; ICU: Intensive care unit; EGD: Esophagogastroduodenoscopy; CT: Computerized tomography; CyBorD: Cyclophosphamide, bortezomib, and dexamethasone; AA: Serum amyloid A; MM: Multiple myeloma; ASCT: Autologous stem cell 
transplant; MDex: Melphalan and dexamethasone; CTDa: Cyclophosphamide, thalidomide and dexamethasone.

\section{Acknowledgements}

We would like to thank Dr. Chris MacKnight, Associate Professor, Department of Medicine, Dalhousie University, for review of this manuscript.

\section{Authors' contributions}

RC wrote the manuscript and reviewed the literature. SC edited the manuscript and is the article guarantor. Both authors read and approved the final manuscript.

\section{Funding}

$\mathrm{RC}$ and $\mathrm{SC}$ have no financial disclosures to report.

\section{Availability of data and materials}

Not applicable.

\section{Declarations}

Ethics approval and consent to participate

Not applicable.

\section{Consent for publication}

Informed consent to publish this case report has been obtained in writing from the deceased patient's substitute decision maker.

\section{Competing interests}

The authors declare that they have no competing interests.

\section{Author details}

'Department of Medicine, Dalhousie University and Nova Scotia Health, QEII Health Sciences Centre, VG Site, Suite 442 Bethune Building, 1276 South Park Street, Halifax, NS B3H 2Y9, Canada. ${ }^{2}$ Division of Gastroenterology, Saint John Regional Hospital, 400 University Avenue, Saint John, NB E2L 4L2, Canada.

Received: 23 February 2021 Accepted: 19 July 2021

Published online: 29 July 2021

\section{References}

1. Wuerth BA, Rockey DC. Changing epidemiology of upper gastrointestinal hemorrhage in the last decade: a nationwide analysis. Dig Dis Sci. 2017:62:1286-93.

2. Wechalekar AD, Gillmore JD, Hawkins PN. Systemic amyloidosis. Lancet. 2016;387:2641-54.
3. lida T, Yamano H, Nakase H. Systemic amyloidosis with gastrointestinal involvement: diagnosis from endoscopic and histological views. J Gastroenterol Hepatol. 2018;33(3):583-90.

4. Singh K, Chapalamadugu P, Malet P. Upper gastrointestinal bleeding due to amyloidosis in a patient with multiple myeloma. Clin Gastroenterol Hepatol. 2016;14(11):A22-3.

5. Sundaram S, Rathod R. Gastric amyloidosis causing nonvariceal upper gastrointestinal bleeding. ACG Case Rep J. 2019;6(5):66.

6. Gjeorgjievski M, Purohit T, Amin MB, et al. Upper gastrointestinal bleeding from gastric amyloidosis in a patient with smoldering multiple myeloma. Case Rep Gastrointest Med. 2015. https://doi.org/10.1155/2015/320120.

7. Quock TP, Tingjian Y, Chang E, et al. Epidemiology of AL amyloidosis: a real-world study using US claims data. Blood Adv. 2018;2(10):1046-53.

8. Obici L, Perfettie V, Palladini G, et al. Clinical aspects of systemic amyloid diseases. Biochem Biophys Acta. 2005;1(1753):11-22.

9. Wisniowski B, Wechalekar A. Confirming the diagnosis of amyloidosis. Acta Haematol. 2020;143(4):312-21.

10. Cowan AJ, Skinner M, Seldin DC, et al. Amyloidosis of the gastrointestinal tract: A 13-year, single-center, referral experience. Haematologica. 2013:98(1):141-6.

11. Menke DM, Kyle RA, Fleming CR, et al. Symptomatic gastric amyloidosis in patients with primary systemic amyloidosis. Mayo Clin Proc. 1993;68(8):763-7.

12. Tada S, lida M, Iwashita A, et al. Endoscopic and biopsy findings of the upper digestive tract in patients with amyloidosis. Gastrointest Endosc. 1990;36(1):10-4.

13. James DG, Zuckerman GR, Sayuk GS, et al. Clinical recognition of AL type amyloidosis of the luminal gastrointestinal tract. Clin Gastroenterol Hepatol. 2007:5(5):582-8.

14. Rowe K, Pankow J, Salyers W. Gastrointestinal amyloidosis: review of the literature. Cureus. 2017. https://doi.org/10.7759/cureus.1228.

15. Lim AY, Lee JH, Jung KS, Gwag HB, Kim DH, Kim SJ, Lee GY, Kim JS, Kim HJ, Lee SY, Lee JE. Clinical features and outcomes of systemic amyloidosis with gastrointestinal involvement: a single-center experience. Korean J Intern Med. 2015;30(4):496.

16. Siau K, Elzubeir A, Cooper SC, lqbal T. Amyloidosis: an unusual cause of upper gastrointestinal bleeding. Case Rep. 2016;2016:bcr2016217653.

17. Khan Z, Darr U, Renno A, Tiwari A, Sofi A, Nawras A. Massive upper and lower Gl bleed from simultaneous primary (AL) amyloidosis of the stomach and transverse colon in a patient with multiple myeloma. Case Rep Gastroenterol. 2017;11(3):633-9.

\section{Publisher's Note}

Springer Nature remains neutral with regard to jurisdictional claims in published maps and institutional affiliations.
Ready to submit your research? Choose BMC and benefit from:

- fast, convenient online submission

- thorough peer review by experienced researchers in your field

- rapid publication on acceptance

- support for research data, including large and complex data types

- gold Open Access which fosters wider collaboration and increased citations

- maximum visibility for your research: over 100M website views per year

At BMC, research is always in progress.

Learn more biomedcentral.com/submissions 